\title{
CCL21 as an independent favorable prognostic factor for stage III/IV colorectal cancer
}

\author{
YIFENG ZOU $^{1 *}$, YUFENG CHEN $^{1 *}$, XIANRUI WU $^{1}$, RUIXUE YUAN $^{2}$, ZERONG CAI $^{1}$, \\ XIAOSHENG HE ${ }^{1}$, XINJUAN FAN ${ }^{2}$, LEI WANG ${ }^{1,2}$, XIAOJIAN WU ${ }^{1,2}$ and PING LAN ${ }^{1,2}$ \\ ${ }^{1}$ Department of Colorectal Surgery, The Sixth Affiliated Hospital of Sun Yat-sen University, Guangzhou; \\ ${ }^{2}$ Gastrointestinal Institute of Sun Yat-sen University, Guangzhou, Guangdong, P.R. China
}

Received February 11, 2013; Accepted March 28, 2013

DOI: $10.3892 /$ or.2013.2533

\begin{abstract}
The aim of the present study was to investigate the expression dynamics of CCL21 and its prognostic significance in human stage III/IV colorectal cancer (CRC). CCL21 expression dynamics were detected with western blotting. The expression of CCL21 in CRC tissue microarrays was examined by immunohistochemistry. The optimal cut-point of CCL21 expression was assessed by the X-tile program. The prognostic significance was analyzed using both Kaplan-Meier curves and Cox regression analysis. Western blot analysis demonstrated that CCL21 expression was comparable in the $\mathrm{CRC}$ and normal colorectal tissues. According to the X-tile program, the cut-point for high expression of CCL21 in CRC was determined when the CCL21 expression index was $>56.1$. Overexpression of CCL21 was significantly correlated with larger tumor diameter, more mucinous carcinoma or signet ring cell carcinoma and poor tumor differentiation. Patients with high expression of CCL21 had a higher overall survival rate in comparison to patients with low expression. In the multivariate Cox regression analysis, CCL21 expression was found to be an independent prognostic biomarker for CRC. ROC curves showed that CCL21 expression could improve the prognostic capability of TNM stage in stage III/IV CRC patients. High expression of CCL21 is an independent and useful biomarker for predicting longer survival of stage III/ IV CRC patients.
\end{abstract}

Correspondence to: Professor Ping Lan or Professor Xiaojian Wu, Department of Colorectal Surgery, The Sixth Affiliated Hospital of Sun Yat-Sen University, 26 Yuancun Erheng Road, Guangzhou, Guangdong 510655, P.R. China

E-mail: sumslp@163.com

E-mail:wxjmqy2003@yahoo.com

*Contributed equally

Key words: CCL21, colorectal neoplasms, prognosis

\section{Introduction}

Colorectal cancer (CRC) is the third most common malignancy in males and the second most common in females worldwide, with more than 1.2 million newly diagnosed cases and 608,700 deaths in 2008 (1). In China, the incidence of CRC has been increasing annually in both urban and rural areas since 1998, and it is estimated to rise continuously in the next few years (2). In contrast, the incidence of CRC has been reported to be stabilized in developed countries. Moreover, the prognosis of CRC patients has improved over time $(3,4)$, which is at least partly attributed to the application of advanced treatment modalities, such as individualized treatment. The key to successful individualized treatment lies in the development of reliable biomarkers, which can both guide treatment and help to predict prognosis. However, most of the biomarkers evaluated in previous studies lack the appropriate qualities. Therefore, efforts in identifying suitable biomarkers are still needed.

The tumor immune microenvironment, a hallmark of the tumor, plays an important role in the development as well as the progression of CRC (5). Except for local infiltrating immune cells, chemokines, such as CXCL5 (6) and CAECAM1 (7), have an effect on the growth and metastasis of CRC. CCL21, known as 6Ckine/SLC, is a CC-type chemokine with specificity to CCR7 receptors via a uniquely long C-terminal tail containing 32 amino acids (8). Constitutive expression of CCL21 regulates cell recruitment in homeostasis, particularly in the homing of dendritic cells (DCs) as well as subpopulations of T cells (9). Previous studies have shown that CCL21 plays different roles in different tumors, namely presenting a metastasis-promoting effect on melanoma (10), non-small cell lung cancer (11) and gastric cancer (12), while functioning as a protecting factor in prostate cancer (13). However, the impact of CCL21 expression on the prognosis of CRC patients has not been well defined. Thus, the aim of the present study was to evaluate the prognostic value of CCL21 in a large cohort of stage III/IV CRC patients.

In the present study, a cohort of stage III/IV CRC patients with a long-term follow-up was enrolled. CCL21 density was detected using immunohistochemical analysis (IHC) and the TMAJ program. The cohort was divided into two groups based on the optimal cut-point, which was generated by X-tile 
program. The clinical prognostic significance of CCL21 in stage III/IV CRC patients was subsequently analyzed.

\section{Materials and methods}

Patients and cohorts. To detect the expression dynamics of CCL21 in stage III/IV CRC patients, 12 paired freshly frozen specimens from primary CRC tissues and normal colorectal tissues were obtained from the Tissue Bank of the Sixth Affiliated Hospital of Sun Yat-Sen University (Guangzhou, China). All the samples were confirmed histologically.

To detect the association between the expression of CCL21 and the prognosis of patients, 143 CRC patients with TNM stage III/IV disease, who underwent initial resection at the First Affiliated Hospital of Sun Yat-Sen University (Guangzhou, China) from 2000 to 2005, were enrolled for tissue microarray construction. Patients who received preoperative chemotherapy and/or radiotherapy were excluded from the study. Clinicopathological variables collected from the database included age, gender, body mass index (BMI), history of smoking and alcohol consumption, family history of cancer, preoperative bowel obstruction, preoperative serum CEA and CA199 levels, tumor location, tumor diameter, histopathology, degree of differentiation, depth of tumor invasion, nodal status and American Joint Committee on Cancer (AJCC) stage (7th edition). This study was approved by the Institutional Review Board (IRB) of Sun Yat-Sen University (Guangzhou, China).

Patient follow-up was carried out according to an original plan by two specialists. All CRC patients were evaluated at the hospital, by telephone, or mail correspondence, quarterly for the first year, semi-annually for the second year, and annually thereafter. The primary endpoint was overall survival (OS), which was defined as the time interval from surgery to death. The secondary endpoint was disease-free survival (DFS) time, defined as the time interval from surgery to the first event of either recurrent disease or death.

Western blot analysis. Tissue protein extractions were prepared using T-PER tissue protein extraction reagent (Pierce, Rockford, IL, USA) and a protease inhibitor cocktail (SigmaAldrich, St. Louis, MO, USA). For each sample, $30 \mu \mathrm{g}$ of protein was loaded and separated by $10 \%$ SDS-polyacrylamide gel and then transferred to NC membranes (Millipore Corp., Billerica, MA, USA). After blocking with 5\% non-fat dry milk in TBST, membranes were probed overnight at $4^{\circ} \mathrm{C}$ with the CCL21 antibody (1:1,000; Abcam, Cambridge, UK) and the $\beta$-actin antibody (Santa Cruz Europe). The membranes were then washed with TBST 3 times and incubated with the secondary antibodies at room temperature for $1 \mathrm{~h}$ (goat antirabbit IgG-HRP; Santa Cruz Biotechnology). Fluorescence on the membrane was generated using the ECL Plus kit after the membranes were washed 3 times with TBST. Protein densitometry was performed using Gel-Pro Analyzer 4.0 software (Media Cybernetics, Inc., Silver Spring, MD, USA), and CCL21 expression was normalized against $\beta$-actin.

Tissue microarray construction and immunohistochemical analysis. In the tissue microarray construction, two cores of tissue were punched from the representative tumor areas and deposited into a recipient block with the tissue array instrument, MiniCore (Alphelys, Paris, France), and 5- $\mu \mathrm{m}$ sections were cut for immunohistochemical staining. All slides were deparaffinized and rehydrated through graded alcohols. Endogenous peroxidase was blocked with $0.3 \%$ hydrogen peroxide for $10 \mathrm{~min}$ at room temperature before the slides were exposed to the antigen retrieval system $(10 \mathrm{mM}$ sodium citrate, $0.05 \%$ Tween-20, $\mathrm{pH} 6.0$ for $25 \mathrm{~min}$ ). After incubation with the primary rabbit multiclonal CCL21 antibody (1:500; Abcam) overnight at $4^{\circ} \mathrm{C}$, the sections were stained with diaminobenzidine in an EnVision System (DakoCytomation, Glostrup, Denmark). The slides were finally counterstained with hematoxylin (Beijing Zhongshan Golden Bridge Biotechnology Co., Ltd., Beijing, China).

Evaluation of immunohistochemical (IHC) analysis. A digital image (2592x1944 pixels, 13.9 nanometers/pixel) for each tumor spot was captured (fold magnification, x200) with a Leica DMI4000B microscope (Leica Microsystems, Wetzlar, Germany). The CCL21 expression index was then estimated in the TMAJ project (Johns Hopkins TMA Core Facility, Baltimore, MD, USA), calculated as the product of CCL21 staining intensity and the density. The intensity was provided by the software automatically, and the density was the quotient between the positive area and total area (Fig. 1C). Finally, the mean IHC index for each patient was used for statistical analysis without knowledge of the clinicopathological information. The median CCL21 expression index was 7.2 (range, 0-187.7).

Selection of the cut-point value. Cut-point of the CCL21 expression index was optimized with the X-tile program (version 3.6.1, Yale University School of Medicine, New Haven, CT, USA) based on CRC patient outcomes (14). In the X-tile program, the cohort was divided randomly into a matched training and validation sets, as a method for the optimal cut-point selection. The cut-point value derived from the training set was then parsed in the validation set, and statistical significance was determined by using a standard log-rank method, while correcting for the use of minimum $\mathrm{P}$ statistics by Miller-Siegmund P-value correction (15).

Statistical analysis. The expression dynamics of CCL21 between CRC and normal colorectal tissues was evaluated using the paired t-test. Correlations between the CCL21 expression index and clinicopathological characteristics were analyzed using the 2-tail t-test (or Wilcoxon rank sum test as appropriate) for continuous variables and the Chi-square test (or the Fisher's exact test as appropriate) for categorical variables. Patient survival was depicted using Kaplan-Meier curves with a log-rank test. Both univariate and multivariate analyses were performed to evaluate the risk factors for both OS and DFS in the stage III/IV CRC patients. Receiver operating characteristic (ROC) curve analysis was carried out to assess the predictive value of the parameters, including a combined parameter, which was defined as the combination of the CCL21 expression index and TNM stage, ranging from 0 to 2 . All statistical analyses were performed using the SPSS software, version 16 (SPSS, Inc., Chicago, IL, USA). P-value $<0.05$ was considered to indicate a statistically significant result. 

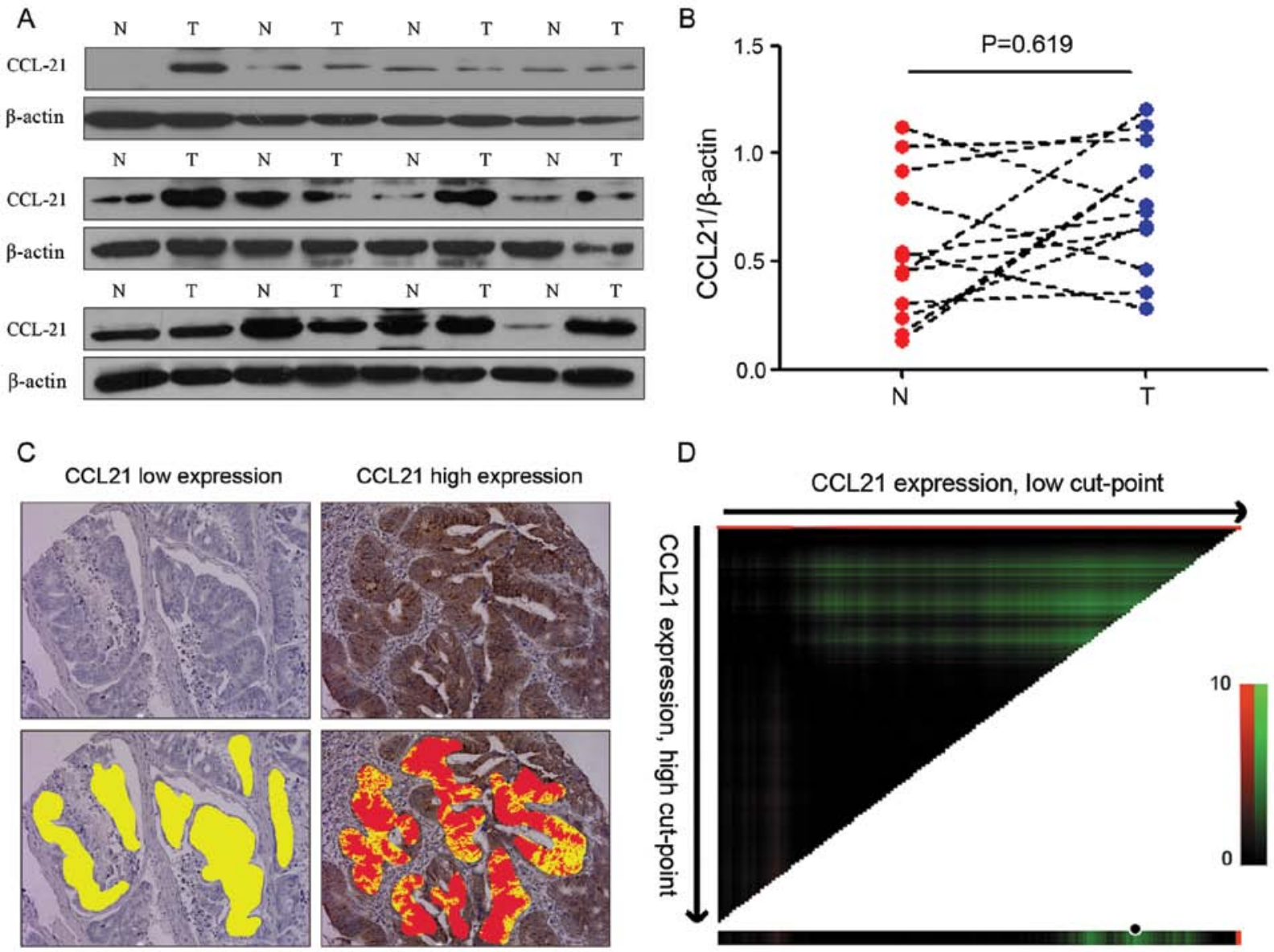

Figure 1. CCL21 expression dynamics in CRC and normal colorectal tissues and immunohistochemical staining and evaluation of CCL21 expression in CRC. (A) Western blot analysis of CCL21 expression in 12 paired CRC and normal colorectal tissues was performed. Expression levels of CCL21 were normalized with those of $\beta$-actin. (B) CCL21 expression levels in paired tissues were compared using a paired t-test, which did not indicate a significant difference between the $\mathrm{CRC}$ and normal colorectal tissues ( $\mathrm{P}=0.619)$. (C) Representative examples of the different levels of CCL21 expression are shown (upper panels), and digital images were analyzed using the TMAJ software (fold magnification, x200) (bottom panels). The yellow area is defined as the total area analyzed, while the red area is the positive area. (D) Optimal cut-point was determined using the X-tile program. The CCL21 expression index is 2.8 (range, 0-56.1) and 109.8 (range, 56.1-187.7) for the low and high expression samples, respectively.

\section{Results}

Patient demographics and follow-up. A total of 143 patients with stage III/IV CRC met the inclusion criteria and were enrolled for immunohistochemical analysis. Seventy-eight patients $(54.5 \%)$ were male and $65(45.5 \%)$ were female, with a mean age of $56.7 \pm 14.5$ years. Based on the 7 th AJCC classification, there were 114 stage III patients and 29 stage IV patients. Site of cancer was the rectum in 72 patients $(50.3 \%)$ and the colon in 71 patients (49.7\%). After a mean follow-up of $49.5 \pm 30.6$ months, 59 patients either died or had disease recurrence.

CCL21 expression dynamics and selection of the optimal cut-point value. Regarding the expression dynamics, western blot analysis revealed that CCL21 expression was comparable between the tumor tissue and the normal colorectal specimens ( $\mathrm{P}=0.619$, Fig. $1 \mathrm{~A}$ and $\mathrm{B})$. CCL21 expression in all $143 \mathrm{CRC}$ patients was evaluated through tissue microarrays, and the index of CCL21 expression was calculated using the TMAJ project (Fig. 1C). The CCL21 expression index ranged from 0 to 187.7 (median 7.2). According to the X-tile program, patients were divided into a low expression group and a high expression group based on a cut-point of 56.1 $(\mathrm{P}=0.022$, Fig. 1D).

Association between CCL21 expression, clinicopathological variables and patient survival. High expression of CCL21 was identified in 27 (18.9\%) of 143 stage III/IV CRC patients (Table I). The tumor diameter was significantly larger in patients with high CCL21 expression than in those with low expression $(\mathrm{P}=0.007)$. Patients with high CCL21 expression were also found to have more mucinous or signet ring cell carcinoma $(\mathrm{P}<0.001)$ and poor tumor differentiation $(\mathrm{P}=0.001)$. However, no significant difference was identified between patients with high and low expression in terms of the other clinicopathological variables $(\mathrm{P}>0.05)$.

Both Kaplan-Meier analysis and univariate Cox proportional hazard regression model were applied to investigate the impact of CCL21 overexpression on stage III/IV CRC patient survival. High expression of CCL21 was significantly correlated with better overall survival (log-rank, $\mathrm{P}=0.022$ ) (Fig. $2 \mathrm{~A}$ and Table II). Additional clinicopathological variables, including preoperative serum CEA level $(\mathrm{P}=0.001)$, preoperative serum CA199 level $(\mathrm{P}=0.003)$, nodal status $(\mathrm{P}=0.006)$ and distant metastasis $(\mathrm{P}<0.001)$, were also found to be significant 
Table I. Clinicopathological characteristics of the 143 stage III/IV CRC patients.

\begin{tabular}{|c|c|c|c|c|}
\hline Characteristics & All cases & $\begin{array}{l}\text { Low CCL21 } \\
\text { expression }\end{array}$ & $\begin{array}{l}\text { High CCL21 } \\
\text { expression }\end{array}$ & P-value ${ }^{a}$ \\
\hline No. of patients & 143 & 116 & 27 & \\
\hline Mean age (years) & $56.7 \pm 14.5$ & $57.0 \pm 15.0$ & $55.6 \pm 12.5$ & $0.654^{\mathrm{b}}$ \\
\hline Gender, n (\%) & & & & 0.160 \\
\hline Male & 78 & $60(51.7)$ & $18(66.7)$ & \\
\hline Female & 65 & $56(48.3)$ & $9(33.3)$ & \\
\hline $\mathrm{BMI}, \mathrm{kg} / \mathrm{m}^{2}$ & $21.1 \pm 3.2$ & $20.8 \pm 3.1$ & $22.1 \pm 3.5$ & $0.075^{\mathrm{b}}$ \\
\hline Bowel obstruction, n (\%) & & & & $0.730^{\mathrm{c}}$ \\
\hline Yes & 14 & $11(9.6)$ & $3(11.1)$ & \\
\hline No & 128 & $104(90.4)$ & $24(88.9)$ & \\
\hline CEA (ng/ml), n (\%) & & & & 0.659 \\
\hline$<5$ & 74 & $59(55.1)$ & $15(60.0)$ & \\
\hline$\geq 5$ & 58 & 48 (44.9) & $10(40.0)$ & \\
\hline CA199 (ng/ml), n (\%) & & & & 0.331 \\
\hline$<37.5$ & 89 & $74(72.5)$ & $15(62.5)$ & \\
\hline$\geq 37.5$ & 37 & $28(27.5)$ & $9(37.5)$ & \\
\hline Tumor location, n (\%) & & & & 0.496 \\
\hline Colon & 71 & $56(48.3)$ & $15(55.6)$ & \\
\hline Rectum & 72 & $60(51.7)$ & $12(44.4)$ & \\
\hline Tumor diameter, $\mathrm{cm}$ & $5.1 \pm 2.2$ & $4.9 \pm 2.1$ & $6.1 \pm 2.5$ & $0.007^{b}$ \\
\hline Histopathology, n (\%) & & & & $<0.001$ \\
\hline Adenocarcinoma & 126 & $110(94.8)$ & $16(59.3)$ & \\
\hline Mucinous carcinoma or signet ring cell carcinoma & 17 & $6(5.2)$ & $11(40.7)$ & \\
\hline Differentiation, n (\%) & & & & 0.001 \\
\hline Well/moderate & 114 & $99(85.3)$ & $15(55.6)$ & \\
\hline Poor & 29 & $17(14.7)$ & $12(44.4)$ & \\
\hline Depth of tumor invasion, $\mathrm{n}(\%)$ & & & & $1.000^{\mathrm{c}}$ \\
\hline $\mathrm{T} 1+\mathrm{T} 2$ & 10 & $8(6.9)$ & $2(7.4)$ & \\
\hline $\mathrm{T} 3+\mathrm{T} 4$ & 133 & $108(93.1)$ & $25(92.6)$ & \\
\hline Nodal status, n (\%) & & & & $0.675^{\mathrm{c}}$ \\
\hline N0 & 9 & $7(6.2)$ & $2(7.7)$ & \\
\hline $\mathrm{N} 1+\mathrm{N} 2$ & 130 & $106(93.8)$ & $24(92.3)$ & \\
\hline Distant metastasis, $\mathrm{n}(\%)$ & & & & 0.800 \\
\hline M0 & 114 & $92(79.3)$ & $22(81.5)$ & \\
\hline M1 & 29 & $24(20.7)$ & $5(18.5)$ & \\
\hline
\end{tabular}

P-value as calculated by ${ }^{a}$ Chi-square test, ${ }^{b}$-test and ${ }^{c}$ Fisher's exact test.

prognostic factors for patient overall survival (Table II). Similar results were also identified for patient disease-free survival (Fig. 2B and Table II). Of note, after being stratified by the tumor location, CCL21 expression was still found to have an impact on patient survival (OS, $\mathrm{P}=0.025$; $\mathrm{DFS}, \mathrm{P}=0.020)$.

CCL21 expression as a significant prognostic factor in the multivariate analysis. As expected, high expression of CCL21 remained a significant independent prognostic factor for favorable overall survival in the multivariate analysis after adjusting for preoperative serum CEA level, preoperative serum CA199 level, nodal status and distant metastasis [hazard ratio (HR), 10.204; 95\% confidence interval (CI) , 2.358-45.455; $\mathrm{P}=0.002$; Table III]. As expected, analysis of disease-free survival showed similar results (HR, 9.524; 95\% CI, 2.232-40.000; $\mathrm{P}=0.002$; Table III).

ROC curve analysis was also applied to further assess the prognostic value of CCL21 expression, which showed an encouraging role of CCL21 expression in predicting CRC patient overall survival and disease-free survival [area under 
Table II. Univariate analyses of CCL21 expression and clinicopathological variables in the 143 stage III/IV CRC patients.

\begin{tabular}{|c|c|c|c|c|}
\hline \multirow[b]{2}{*}{ Characteristics } & \multicolumn{2}{|c|}{ Overall survival } & \multicolumn{2}{|c|}{ Disease-free survival } \\
\hline & $\mathrm{HR}(95 \% \mathrm{CI})$ & P-value ${ }^{a}$ & HR $(95 \% \mathrm{CI})$ & P-value \\
\hline Age (years) & & 0.257 & & 0.298 \\
\hline$<56.7^{\mathrm{b}}$ & 1.0 & & 1.0 & \\
\hline$\geq 56.7$ & $1.355(0.801-2.294)$ & & $1.322(0.781-2.237)$ & \\
\hline Gender & & 0.765 & & 0.740 \\
\hline Female & 1.0 & & 1.0 & \\
\hline Male & $0.925(0.554-1.543)$ & & $0.917(0.549-1.530)$ & \\
\hline $\mathrm{BMI}, \mathrm{kg} / \mathrm{m}^{2}$ & & 0.458 & & 0.417 \\
\hline$<21.1^{\mathrm{b}}$ & 1.0 & & 1.0 & \\
\hline$\geq 21.1$ & $0.820(0.487-1.383)$ & & $0.805(0.478-1.358)$ & \\
\hline Bowel obstruction & & 0.515 & & 0.596 \\
\hline No & 1.0 & & 1.0 & \\
\hline Yes & $1.284(0.605-2.724)$ & & $1.225(0.578-2.597)$ & \\
\hline $\mathrm{CEA}(\mathrm{ng} / \mathrm{ml})$ & & 0.001 & & 0.001 \\
\hline$<5$ & 1.0 & & 1.0 & \\
\hline$\geq 5$ & $2.576(1.454-4.567)$ & & $2.720(1.524-4.854)$ & \\
\hline CA199 (ng/ml) & & 0.003 & & 0.004 \\
\hline$<37.5$ & 1.0 & & 1.0 & \\
\hline$\geq 37.5$ & $2.461(1.371-4.417)$ & & $2.387(1.330-4.285)$ & \\
\hline Tumor location & & 0.240 & & 0.271 \\
\hline Colon & 1.0 & & 1.0 & \\
\hline Rectum & $1.362(0.813-2.283)$ & & $1.336(0.798-2.237)$ & \\
\hline Tumor diameter $(\mathrm{cm})$ & & 0.635 & & 0.689 \\
\hline$<5^{\mathrm{b}}$ & 1.0 & & 1.0 & \\
\hline$\geq 5$ & $0.884(0.530-1.473)$ & & $0.901(0.540-1.502)$ & \\
\hline Histopathology & & 0.910 & & 0.985 \\
\hline Adenocarcinoma & 1.0 & & 1.0 & \\
\hline Mucous carcinoma & $0.952(0.408-2.222)$ & & $0.992(0.425-2.314)$ & \\
\hline Differentiation & & 0.071 & & 0.058 \\
\hline Well + moderate & 1.0 & & 1.0 & \\
\hline Poor & $1726(0.955-3.117)$ & & $1.771(0.981-3.199)$ & \\
\hline Depth of invasion & & 0.564 & & 0.589 \\
\hline $\mathrm{T} 1+\mathrm{T} 2$ & 1.0 & & 1.0 & \\
\hline $\mathrm{T} 3+\mathrm{T} 4$ & $1.408(0.440-4.507)$ & & $1.378(0.430-4.413)$ & \\
\hline Nodal status & & 0.006 & & 0.013 \\
\hline N0 & 1.0 & & 1.0 & \\
\hline $\mathrm{N} 1+\mathrm{N} 2$ & $0.329(0.148-0.730)$ & & $0.366(0.165-0.812)$ & \\
\hline Distant metastasis & & $<0.001$ & & $<0.001$ \\
\hline M0 & 1.0 & & 1.0 & \\
\hline M1 & $6.563(3.839-11.218)$ & & $6.214(3.621-10.662)$ & \\
\hline CCL21 expression & & 0.029 & & 0.025 \\
\hline Low & 1.0 & & 1.0 & \\
\hline High & $0.360(0.144-0.901)$ & & $0.349(0.139-0.875)$ & \\
\hline
\end{tabular}

${ }^{a}$ The P-value was calculated using the univariate Cox proportional hazard regression model. ${ }^{\mathrm{b}}$ Mean value. HR, hazard ratio; CI, confidence interval. 
Table III. Cox multivariate analyses of prognostic factors on patient survival.

\begin{tabular}{lccr}
\hline Characteristics & Hazard ratio & 95\% CI & P-value $^{\mathrm{a}}$ \\
\hline Overall survival & & & 0.088 \\
CEA, ng/ml ( $\geq 5$ vs. $<5)$ & 1.783 & $0.917-3.467$ & 0.079 \\
CA199, ng/ml $(\geq 37.5$ vs. $<37.5)$ & 1.793 & $0.934-3.440$ & 0.945 \\
Nodal status (N1+N2 vs. N0) & 0.964 & $0.338-2.747$ & $<0.001$ \\
Distant metastasis (M0 vs. M1) & 11.196 & $5.156-24.313$ & 0.002 \\
CCL21 density (low vs. high) & 10.204 & $2.358-45.455$ & 0.049 \\
Disease-free survival & & & 0.039 \\
CEA, ng/ml ( $\geq 5$ vs. $<5)$ & 1.952 & $1.003-3.797$ & 0.529 \\
CA199, ng/ml ( $\geq 37.5$ vs. $<37.5)$ & 1.977 & $1.034-3.781$ & $<0.001$ \\
Nodal status (N1+N2 vs. N0) & 1.396 & $0.494-3.939$ & 0.002 \\
Distant metastasis (M0 vs. M1) & 11.574 & $5.300-25.275$ & $2.232-40.000$ \\
CCL21 density (low vs. high) & 9.524 & &
\end{tabular}

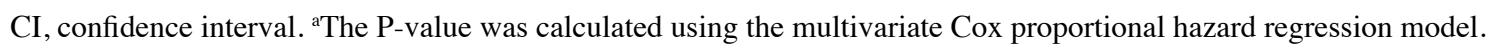

A

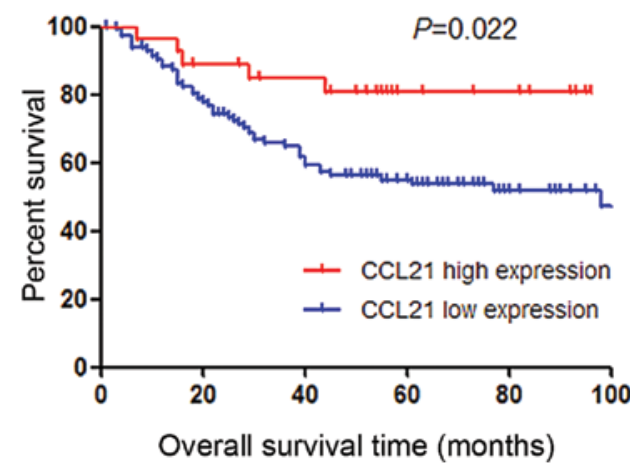

B

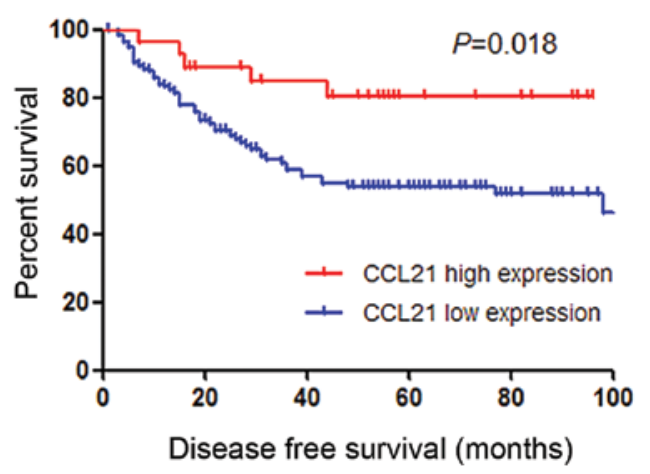

Figure 2. Kaplan-Meier curves stratified by CCL21 expression in 143 stage III/IV CRC patients. High expression of CCL21 was significantly correlated with better survival, both for overall survival (left) and disease-free survival (right) ( $\mathrm{P}=0.022$ and 0.018 , respectively).

\section{ROC curve}
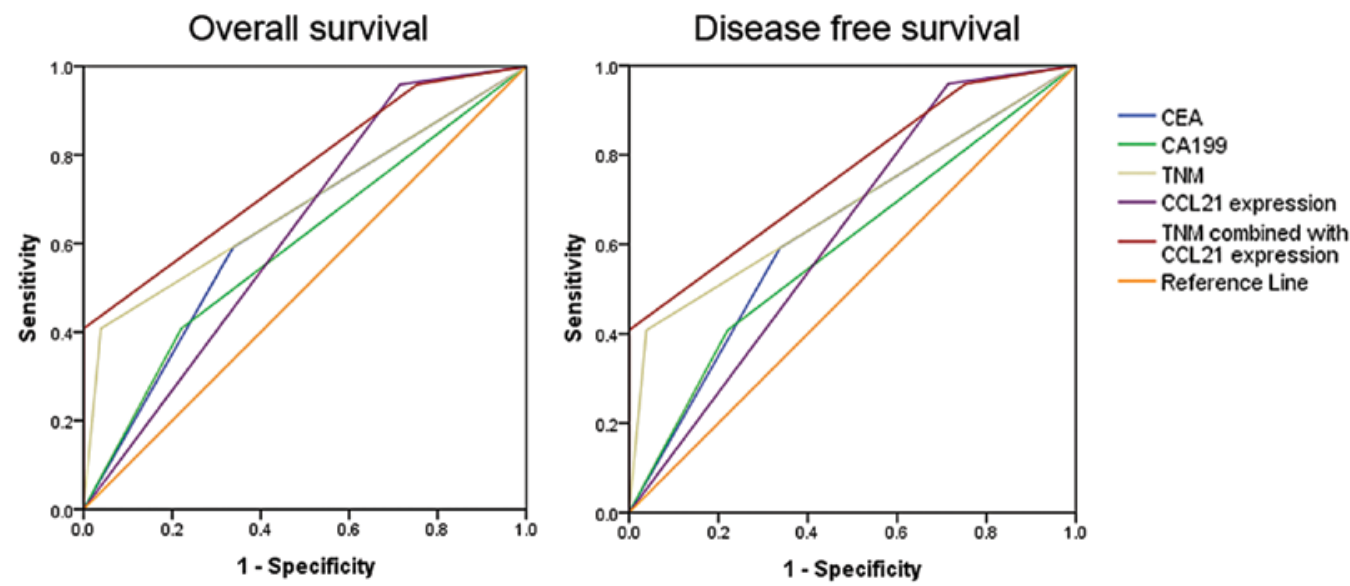

Figure 3. ROC curves of the clinicopathological variables and CCL21 expression based on the outcomes of 143 stage III/IV CRC patients. Overall survival (left): TNM combined with CCL21 expression [area under the curve (AUC) $=0.757 ; \mathrm{P}<0.001$ ], CCL21 expression (AUC=0.622; $\mathrm{P}=0.021$ ), TNM (AUC=0.685; $\mathrm{P}<0.001$ ), $\mathrm{CA} 199$ (AUC=0.594; $\mathrm{P}=0.077$ ) and CEA ( $\mathrm{AUC}=0.627 ; \mathrm{P}=0.016$ ). Disease-free survival (right): TNM combined with CCL21 expression $[$ area under the curve $(\mathrm{AUC})=0.757 ; \mathrm{P}<0.001]$, CCL21 expression (AUC=0.622; $\mathrm{P}=0.021)$, TNM (AUC=0.685; $\mathrm{P}<0.001)$, CA199 (AUC=0.594; $\mathrm{P}=0.077)$ and CEA (AUC=0.627; $\mathrm{P}=0.016)$. 
the curve $(\mathrm{AUC})=0.622$ and $0.622 ;$ Fig. 3]. More importantly, the AUC value was increased after incorporating CCL21 expression into TNM stage (AUC=0.757 and 0.757, Fig. 3).

\section{Discussion}

Despite the improved diagnostic techniques and advanced treatments, CRC remains a major public health issue. Due to the limitations of the current AJCC staging system (16), numerous studies in the literature have evaluated the prognostic roles of biomarkers for CRC patients $(17,18)$. In vitro, CCL21 was found to be able to promote the invasive ability of colorectal cancer cells by inducing MMP-9 expression (19). However, CCL21 was also shown to enhance the infiltration of immune cells into the tumor region, inhibiting the growth of tumors by generating an antitumor cellular immune response (20,21). Although the prognostic value of CCL21 has been studied in several types of cancers, including gastric (12) and pancreatic cancer (20), its role in CRC is not well defined. Mumtaz et al (22) found that the expression of CCL21 was decreased in rectal cancer, but the prognostic value of CCL21 was not further evaluated due to the lack of appropriate followup.

In the present study, western blot analysis of 12 paired freshly frozen specimens from primary stage III/IV CRC and normal colorectal tissues was performed to examine the expression dynamics of CCL21. In contrast with a previous study (22), CCL21 expression was not found to be significantly different from that in normal colorectal tissues in our study. This might be explained by the nature of the heterogeneity of CRCs, which results in varied expression of CCL21 in different CRC patient tumors. To avoid a predetermined arbitrary cut-point, we constructed X-tile plots for assessment of scores which divide CCL21 expression into two populations, in which we corrected for the use of minimum P statistics by Miller-Siegmund P-value correction (15). In the present study, our results revealed that CCL21 expression in CRC was closely associated with tumor diameter, tumor histology and tumor differentiation. In this perspective, CCL21 expression may increase as tumor progress. Furthermore, survival analysis revealed that stage III/IV CRC patients with high expression of CCL21 had a prolonged survival, even after being stratified by the tumor location. In the multivariate analyses, CCL21 expression was proven to be a prognostic factor independent of certain well-established clinicopathologcial parameters. CCL21 expression may constitute a useful biomarker in addition to the AJCC TNM staging system for these patients, and identify patients whose tumors are more likely to progress and recur and who are good candidates to receive more aggressive treatment.

Although the underlying mechanisms of the predictive value of CCL21 for stage III/IV CRC patients were not further explored in the present study, there were several lines of evidence to support our findings. The tumor microenvironment consists of cancer cells, immune inflammatory cells, endothelial cells as well as other elements (5). CCL21 may inhibit tumor progression through inducing the function of immune inflammatory cells and endothelial cells. Previous research demonstrated that the antitumor activity of CCL21 was mediated by enhancing the infiltration of mature dendritic cells (DCs) and $\mathrm{CD}^{+} \mathrm{T}$ cells to the tumor (23-25). Further data also suggest that modification of the tumor immune microenvironment may lead to an effective tumor-specific response. Inside, DCs are highly effective at secretion of IL-12 and interferon- $\gamma$, and are thus expected to be beneficial for efficient local antitumor responses, as well as T, NK and NKT cells $(26,27)$. CCL21 is not only important in recruiting DCs and $\mathrm{T}$ cells. It has been classified as a CC chemokine, which binds to the CCR7 receptor, but it also has been shown to bind to the CXC chemokine receptor CXCR3 (28). Therefore, the angiostatic activity of CCL21 by binding to the CXCR3 receptor may also be important in its antitumor capability. CXCR3 is expressed on human microvascular endothelial cells under special conditions, and engagement of CXCR3 by CCL21 may inhibit endothelial cell proliferation in vitro (29). Furthermore, T cells expressing CXCR3 were found to be actively recruited into the invasive margin of CRC and induce the Th1-shifted cellular immune responses (30).

The prognostic value of CCL21 in cancer patients appears to be dependent on tumor type. Although a recent study showed that overexpression of CCL21 was a negative prognostic factor for gastric cancer (12), it was shown to be a favorable prognostic factor for pancreatic and breast cancer (20). Wu et al (31) investigated the effect of exogenous CCL21 expression in breast cancer MCF-7 cells on human monocyte-derived DCs. Overexpression of CCL21 improved the immunogenicity of tumor cells and the function of DCs in migration, antigen uptake and presentation, giving rise to increased Th 1 type cytokine, transformation of perforin-forming $\mathrm{CD} 8^{+} \mathrm{T}$ cells and final $\mathrm{T}$ cell-associated clearance of tumor cells. Due to the immune regulatory and angiostatic effect, overexpression of CCL21 inhibits the progression of CRCs and can be used as a favorable prognostic factor. Moreover, expression of CCL21 may enhance the predictive capability of TNM staging. When combined with CCL21 expression, TNM staging showed a more prominent predictive capability. The possible reason is that the combination of clinicopathological and molecular parameters may be a better representation of tumor characteristics and prognosis.

To the best of our knowledge, the present study is the first to evaluate the prognostic significance of CCL21 expression in stage III/IV CRC patients in a large cohort. Our study revealed that high expression of CCL21 was an independent predictor of more favorable survival, and CCL21 analysis may be used as an additional parameter to TNM staging in predicting patient survival outcomes and in guiding treatment. One limitation of our study was the small cohort of paired tissues for analysis of CCL21 expression dynamics.

In conclusion, in the present study of a large cohort of stage III/IV CRC patients, we provide evidence that high expression of CCL21 is a strong and independent predictor of prolonged survival. Examination of CCL21 expression by IHC analysis can be used as an additional and effective way to identify patients at high risk for tumor progression, thus optimizing individualized treatment for stage III/IV CRC patients.

\section{Acknowledgements}

This study was partially sponsored by the National Natural Science Foundation of China (nos. 91029702 and 81072046), 
the Medical Scientific Research Foundation of Guangdong Province, China (no. WSTJJ20101107445221197902271012) and the Young Teachers Training Program Foundation by Sun Yat-Sen University, China (no. 10ykpy01).

\section{References}

1. Jemal A, Bray F, Center MM, et al: Global cancer statistics. CA Cancer J Clin 61: 69-90, 2011.

2. Dai Z, Zheng RS, Zou XN, et al: Analysis and prediction of colorectal cancer incidence trend in China. Zhonghua Yu Fang Yi Xue Za Zhi 46: 598-603, 2012 (In Chinese).

3. Center MM, Jemal A, Smith RA, et al: Worldwide variations in colorectal cancer. CA Cancer J Clin 59: 366-378, 2009.

4. Edwards BK, Ward E, Kohler BA, et al: Annual report to the nation on the status of cancer, 1975-2006, featuring colorectal cancer trends and impact of interventions (risk factors, screening, and treatment) to reduce future rates. Cancer 116: 544-573, 2010

5. Hanahan D and Weinberg RA: Hallmarks of cancer: the next generation. Cell 144: 646-674, 2011

6. Kawamura M, Toiyama Y, Tanaka K, et al: CXCL5, a promoter of cell proliferation, migration and invasion, is a novel serum prognostic marker in patients with colorectal cancer. Eur J Cancer 48: 2244-2251, 2012.

7. Arabzadeh A, Chan C, Nouvion AL, et al: Host-related carcinoembryonic antigen cell adhesion molecule 1 promotes metastasis of colorectal cancer. Oncogene 32: 849-860, 2012.

8. Yoshida R, Nagira M, Kitaura M, et al: Secondary lymphoidtissue chemokine is a functional ligand for the $\mathrm{CC}$ chemokine receptor CCR7. J Biol Chem 273: 7118-7122, 1998.

9. Rot A and von Andrian UH: Chemokines in innate and adaptive host defense: basic chemokinese grammar for immune cells. Annu Rev Immunol 22: 891-928, 2004.

10. Takeuchi H, Fujimoto A, Tanaka M, et al: CCL21 chemokine regulates chemokine receptor CCR7 bearing malignant melanoma cells. Clin Cancer Res 10: 2351-2358, 2004.

11. Koizumi K, Kozawa Y, Ohashi Y, et al: CCL21 promotes the migration and adhesion of highly lymph node metastatic human non-small cell lung cancer Lu-99 in vitro. Oncol Rep 17: 1511-1516, 2007.

12. Hwang TL, Lee LY, Wang CC, et al: CCL7 and CCL21 overexpression in gastric cancer is associated with lymph node metastasis and poor prognosis. World J Gastroenterol 18 : 1249-1256, 2012.

13. Yousefieh N, Hahto SM, Stephens AL, et al: Regulated expression of CCL21 in the prostate tumor microenvironment inhibits tumor growth and metastasis in an orthotopic model of prostate cancer. Cancer Microenviron 2: 59-67, 2009.

14. Camp RL, Dolled-Filhart M and Rimm DL: X-tile: a new bioinformatics tool for biomarker assessment and outcome-based cut-point optimization. Clin Cancer Res 10: 7252-7259, 2004.

15. Raeside DE: Monte Carlo principles and applications. Phys Med Biol 21: 181-197, 1976.

16. O'Connell JB, Maggard MA and Ko CY: Colon cancer survival rates with the new American Joint Committee on Cancer sixth edition staging. J Natl Cancer Inst 96: 1420-1425, 2004
17. Huang Y, Li W, Chu D, et al: Overexpression of matrix metalloproteinase-21 is associated with poor overall survival of patients with colorectal cancer. J Gastrointest Surg 15: 1188-1194, 2011.

18. Lin KY, Tai C, Hsu JC, et al: Overexpression of nuclear protein kinase CK2 alpha catalytic subunit (CK2alpha) as a poor prognosticator in human colorectal cancer. PLoS One 6: e17193, 2011.

19. Sun RH, Wang GB, Li J, et al: Role of CCL21/CCR7 in invasion of colorectal carcinoma cell line SW480. Ai Zheng 28: 708-713, 2009 (In Chinese).

20. Turnquist HR, Lin X, Ashour AE, et al: CCL21 induces extensive intratumoral immune cell infiltration and specific anti-tumor cellular immunity. Int J Oncol 30: 631-639, 2007.

21. Sharma S, Stolina M, Luo J, et al: Secondary lymphoid tissue chemokine mediates $\mathrm{T}$ cell-dependent antitumor responses in vivo. J Immunol 164: 4558-4563, 2000.

22. Mumtaz M, Wagsater D, Lofgren S, et al: Decreased expression of the chemokine CCL21 in human colorectal adenocarcinomas. Oncol Rep 21: 153-158, 2009.

23. Kirk CJ, Hartigan-O'Connor D, Nickoloff BJ, et al: T celldependent antitumor immunity mediated by secondary lymphoid tissue chemokine: augmentation of dendritic cell-based immunotherapy. Cancer Res 61: 2062-2070, 2001.

24. Nomura T, Hasegawa H, Kohno M, et al: Enhancement of antitumor immunity by tumor cells transfected with the secondary lymphoid tissue chemokine EBI-1-ligand chemokine and stromal cell-derived factor-1alpha chemokine genes. Int J Cancer 91: 597-606, 2001

25. Sharma S, Stolina M, Zhu L, et al: Secondary lymphoid organ chemokine reduces pulmonary tumor burden in spontaneous murine bronchoalveolar cell carcinoma. Cancer Res 61: 6406-6412, 2001.

26. Maldonado-Lopez R, Maliszewski C, Urbain J, et al: Cytokines regulate the capacity of $\mathrm{CD} 8 \alpha^{+}$and $\mathrm{CD} 8 \alpha^{-}$dendritic cells to prime Th1/Th2 cells in vivo. J Immunol 167: 4345-4350, 2001.

27. Tatsumi T, Kierstead LS, Ranieri E, et al: Disease-associated bias in T helper type $1(\mathrm{Th} 1) / \mathrm{Th} 2 \mathrm{CD} 4(+) \mathrm{T}$ cell responses against MAGE-6 in HLA-DRB10401(+) patients with renal cell carcinoma or melanoma. J Exp Med 196: 619-628, 2002.

28. Soto H, Wang W, Strieter RM, et al: The CC chemokine 6Ckine binds the CXC chemokine receptor CXCR3. Proc Natl Acad Sci USA 95: 8205-8210, 1998.

29. Romagnani P, Annunziato F, Lasagni L, et al: Cell cycle-dependent expression of CXC chemokine receptor 3 by endothelial cells mediates angiostatic activity. J Clin Invest 107: 53-63, 2001.

30. Musha H, Ohtani H, Mizoi T, et al: Selective infiltration of $\mathrm{CCR}^{+} \mathrm{CXCR}^{+} \mathrm{T}$ lymphocytes in human colorectal carcinoma. Int J Cancer 116: 949-956, 2005.

31. Wu S, Xing W, Peng J, et al: Tumor transfected with CCL21 enhanced reactivity and apoptosis resistance of human monocyte-derived dendritic cells. Immunobiology 213: 417-426, 2008. 